\title{
Scintillator Developments for High Energy Physics and Medical Imaging
}

\author{
P. Lecoq ${ }^{1}$, M. Korzhik ${ }^{2}$ \\ ${ }^{1}$ CERN, Geneva Switzerland \\ ${ }^{2}$ INP, Minsk, Belarus
}

\begin{abstract}
Scintillating crystals have been for a long time developed as a basic component in particle detectors with a strong spinoff in the field of medical imaging. A typical example is $\mathrm{BGO}$, which has become the main component of PET scanners since the large effort made by the L3 experiment at CERN to develop low cost production methods for this crystal.

Systematic R\&D on basic mechanism in inorganic scintillators, initiated by the Crystal Clear Collaboration at CERN 10 years ago, has contributed not to a small amount, to the development of new materials for High Energy Physics and for a new generation of medical imaging devices with increased resolution and sensitivity. The examples of the Lead Tungstate crystal for the CMS experiment at CERN (High Energy Physics) as well as its optimization for possible medical imaging applications will be described with an emphasis on the mutual benefit both fields can extract from a common R\&D effort.
\end{abstract}

\section{INTRODUCTION}

When R. Hofstadter [1] introduced in 1948 Thallium doped Sodium Iodide, $\mathrm{NaI}(\mathrm{Tl})$, he probably did not realize that it was going to be the most popular scintillator for the next 35 years. Unfortunately Sodium Iodide suffers from a relatively low density $\left(3.67 \mathrm{~g} / \mathrm{cm}^{3}\right)$ which limits the spatial resolution and the image quality in medical imaging devices. This is why the discovery of attractive scintillation properties in Bismuth Germanate (BGO) by Weber\&Monchamp in 1973 [2] was immediately recognized for its potential for $\gamma$ cameras, because of its very high density $\left(7.13 \mathrm{~g} / \mathrm{cm}^{3}\right)$. But systematic use of BGO in medical imaging could only be made possible through the large effort of the L3 collaboration at the CERN LEP collider, to develop low cost mass production technology for the $12^{\prime} 000 \mathrm{BGO}$ crystals $\left(1.5 \mathrm{~m}^{3}\right)$ of the $\mathrm{L} 3$ experiment [3]. Today BGO represents more than 50\% of the PET (Positron Emission Tomograph) market.

The need for high density but much faster scintillators for the new generation of hadron colliders triggered a large $R \& D$ effort worldwide in which the Crystal Clear collaboration at CERN [4] has played a major role since 1989. New crystals have been studied, like Lead Tungstate (PWO) and Cerium Fluoride (CeF3). Moreover systematic study of the physics underlying the fundamental scintillation mechanisms has led to a much better prediction capability in the search for new scintillating materials, as it is explained in the report [5] and its 261 references summarizing the Crystal Clear work in the last 5 years. New ultra dense, very fast and efficient scintillators are being studied. Some of them have a high potential for medical imaging devices and are now in the phase of the mass production technology development. A typical example is the one of Lead Tungstate crystals.

After the first announcement and presentation of Lead Tungstate crystal (PWO) at the CRYSTAL 2000 conference in Chamonix at the end of 1992 [6], the CMS collaboration has undertaken in 1994 an ambitious R\&D program on this crystal for its electromagnetic calorimeter at the LHC collider [7]. All the parameters of this crystal have been extensively studied in order to optimize its performances in the context of the LHC collider. As explained in section II, large statistical samples of full size crystals $\left(23 \mathrm{~cm}\right.$ in length, up to $2.5 \times 2.5 \mathrm{~cm}^{2}$ in section) with the desired trapezoidal geometry have been grown and mechanically processed with a yield in excess of $90 \%$. A thorough investigation of the raw material preparation and of the growing conditions has led to a significant improvement in the optical transparency and in the light yield of the crystals. A detailed understanding of the light emission mechanism was the key to solve the problem of the appearance of slow components in some crystals. A systematic analysis of the parameters influencing the structural quality of the crystals was at the origin of a considerable improvement of the radiation hardness of full size crystals. Recently a thorough investigation of the role of different doping ions and the study of their action mechanism in the PWO lattice has led to a significant increase of the light yield of this crystal, which can offer attractive possibilities in medical imaging devices, as explained in section III.

\section{IMPROVEMENT OF PWO PERFORMANCE FOR HIGH ENERGY PHYSICS APPLICATIONS}

In order to meet the tight specifications of the CMS calorimeter [7] and the LHC construction schedule and the CMS running-in in 2005, an ambitious R\&D and production program was organised with the Bogoroditsk TechnoChemical Plant in Russia. The goal is to develop the technology for the cost effective production of full size crystals $(23 \mathrm{~cm}$ in length) with a good light yield of at least 8 $\mathrm{pe} / \mathrm{MeV}$ when measured with a 2 inches Phillips 2262B photomultiplier, in order not to be dominated by statistical fluctuations in the reconstructed energy resolution, a fast decay time of about $20 \mathrm{~ns}$ without contamination by slow components (to avoid pile-up problems), and a very small light yield loss under LHC irradiation of less than 5\% (to allow precise corrections from the monitoring system). Moreover, precise dimensions (tolerances of 10 microns) will guarantee a good hermeticity of the detector. 
After 4 years of very active R\&D significant improvement have been made on the quality of Lead Tungstate crystals. The production has now started and so far more than 1500 crystals of the required quality have been produced in the conditions of mass production.

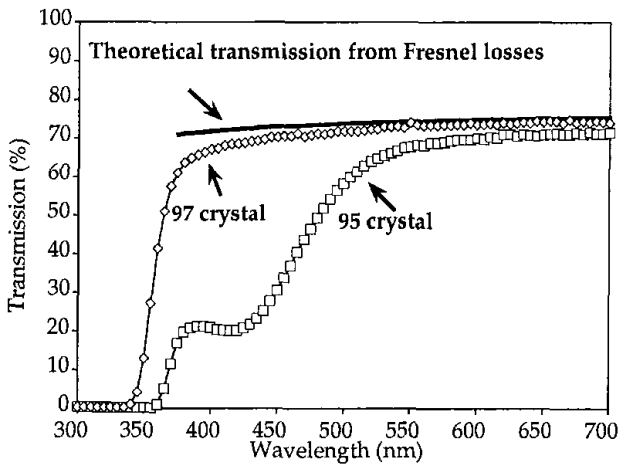

Fig. 1 where the longitudinal optical transmission

The progress on crystal transparency are illustrated on is plotted for two typical crystals, one produced in 95 with a length of $20 \mathrm{~cm}$, and one of the first production batch in September 98 . For comparison the theoretical transmission for a medium with the same index of refraction as PWO is also plotted. A considerable improvement can be seen in the region of scintillation below 500nm, which makes the recent crystals completely colourless. The cleaning of the raw material, particularly for $\mathrm{Mo}, \mathrm{Fe}, \mathrm{Ca}$ improved the crystal transparency in the region $330-370 \mathrm{~nm}$. Moreover the growth of the crystals in an atmosphere poor in oxygen (less than $1 \%$ ) further improved the transparency in the $370-500 \mathrm{~nm}$ range. As a consequence the light yield of the crystals has increased by more than $50 \%$ on average during the same period and reached $12 \mathrm{pe} / \mathrm{MeV}$ (measured with a 2 inches Phillips $2262 \mathrm{~B}$ photomultiplier) for the first production batches. Similarly a systematic investigation of the scintillation mechanism has led to the suppression of all the sources of slow components and delayed luminescence. It was shown for instance that Molybdenum concentration must be lowered to $1 \mathrm{ppm}$ for an efficient suppression of afterglow and slow components $[8,9,10]$.

Since the beginning of the R\&D period the understanding of radiation damage mechanisms in order to improve the PWO radiation hardness has been a major concern. In 1994 we have introduced the doping with pentavalent Niobium ions as an efficient method to suppress di-hole centres [8]. A strong suppression of the $620 \mathrm{~nm}$ induced absorption band is systematically observed with an optimum for a concentration of about $100 \mathrm{ppm}$. But this approach was inactive in the suppression of stable electron centres, generally associated to oxygen vacancies, which also play a role in the radiation damage of PWO. This is why trivalent Yttrium doping has been tested as early as in 1996 on a set of full size crystals. Impressive improvement was observed in test beam, with a light loss saturating at $4 \%$ only for the crystal 1318 in the $\mathrm{H} 4$ test beam at CERN [10]. The effect of doping can nevertheless be modified by other crystal imperfections, like impurities or structural defects. After a systematic cleaning of the raw material a systematic stoechiometry scan was undertaken on more than 30 full size crystals in order to obtain the best suppression of cation and anion vacancies. Two optimum stoechiometric ratio have been obtained corresponding to slightly different structures in PWO [11]. Then a more systematic evaluation of trivalent doping has been made with different tests using Lathanum, Lutetium, Yttrium and Aluminium [10]. The results confirmed those obtained by Kobayashi in the case of Lanthanum, with a significant improvement in the optical transmission edge, scintillation decay and radiation hardness [12].

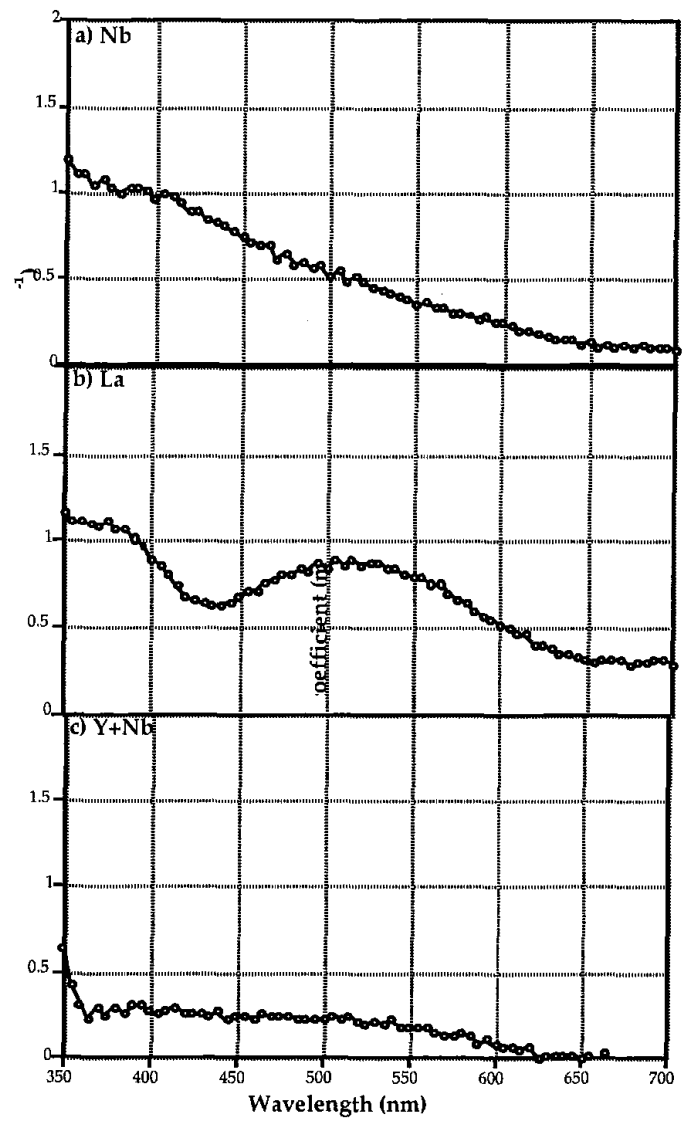

Fig.2: Induced absorption in $23 \mathrm{~cm}$ long PWO crystals with various doping conditions

The relative merits of pentavalent $(\mathrm{Nb})$ and trivalent (mainly La) doping have been discussed in detail in ref [13]. Basically both doping significantly improve the optical transparency and the radiation hardness of crystals. On the other hand, the positive effect of Lathanum doping at high doses looks less clear and the control of the uniformity of the doping is much more difficult for Lanthanum than for Niobium. This has of course implications on the cost of the process. A detailed investigation of the radiation damage mechanisms leads to the conclusion that the ideal situation for Lead Tungstate crystals is a proper tuning of the raw materials, associated to a co-doping. This allows to achieve an 
optimal suppression of both di-hole and electron centres. After several tests the best results have been obtained by doping the crystals with Niobium and Yttrium [13]. One of the reasons Yttrium gives better results than Lathanum for trivalent doping is related to its smaller ionic radius, which leads to a segregation coefficient close to 1 for Yttrium instead of 1.4 for Lanthanum. The positive influence of $\mathrm{Y}$ doping on PWO crystals radiation hardness has been also recently confirmed by another group [14]. This tuning associates all the good features of both Niobium and trivalent ions, without the technological drawbacks associated to Lathanum doping.

In particular there is no problem of damage saturation at high dose as observed on La doped crystals. Fig 2 shows the induced absorption coefficient for 3 crystals ( $\mathrm{Nb}, \mathrm{La}$, and $\mathrm{Y}+\mathrm{Nb}$ co-doped), $23 \mathrm{~cm}$ long, irradiated on the side by a ${ }^{60} \mathrm{Co}$ source at a dose of $500 \mathrm{~Gy}$ and a dose rate of $250 \mathrm{~Gy} /$ hour.

It is to be noticed that the double doping improves by about a factor of two the radiation hardness of the crystals as compared to single doping either with Niobium or Lanthanum. For irradiation conditions similar to LHC a light yield loss of the order of $1 \%$ or less is expected with this optimization. This gives a larger safety factor and significantly improves the yield of acceptable crystals for CMS.

\section{DEVELOPMENTS FOR MEDICAL IMAGING}

There is a need for medical imaging devices for a crystal having the light yield of NaI, the density of BGO, but at least 10 times faster than BGO. A better understanding of the fundamental mechanisms underlying the scintillation properties in many materials has allowed several groups to focus their research on several new scintillators doped with $\mathrm{Ce}^{3+}$ ions, and with a host lattice containing Yttrium, Gadolinum or Lutetium ions. Among the most promising scintillators for a new generation of PET scanners is Luthetium Aluminum Perovskite (LuAP) which has been actively developed by the Crystal Clear collaboration since $1994[15,16,17]$. Another approach looks nevertheless promising, if one can take advantage of the enormous effort to develop low cost mass production technology for High Energy Physics. Crystals can be optimised with adequate performances for medical imaging, but at a much reduced cost as compared to LSO or LuAP. This could open the way for the rapid development of moderate performance but low cost devices which would find applications in early cancer diagnosis for instance. Lead Tungstate is from this point of view an attractive candidate, because of its very high density of 8.28 , fast decay time (about 20ns) and some flexibility to tune optical parameters through specific raw material preparation and doping.

One of the problems of PWO crystals is related to their relatively low light yield at room temperature, which severely limits their use at low energy. An increase of the light yield can be obtained by a redistribution of non radiative losses from the first excited state of regular tungstate oxicomplexes $\mathrm{WO}_{4}{ }^{2-}$. This can be easily achieved by lowering the temperature, or by introducing other radiative centres (for instance with Molybdenum and Lathanum co-doping) with an excitation energy close to the one of regular tungstate groups. This results generally in a deterioration of timing properties of Lead Tungstate with the appearance of slow decay components. But for a correct tuning of the concentration of Mo and La the regular fast scintillation of Lead Tungstate is only slightly modified by a few components in the 100 nsec range. Moreover the emission spectrum is shifted to $500 \mathrm{~nm}$, where the quantum efficiency of most photodetectors is generally better than for the regular emission of Lead Tungstate at $420 \mathrm{~nm}$. Another approach is to make use of the two regular radiating states ${ }^{5} \mathrm{D}_{3},{ }^{5} \mathrm{D}_{4}$ of $\mathrm{Tb}^{3+}$ ion which have an energy less than $27000 \mathrm{~cm}^{-1}$. Upper $\mathrm{f}$ - levels also contribute to the luminescence at $348 \mathrm{~nm}$ at low activator content. The luminescence from ${ }^{5} \mathrm{D}_{3}$ level is strongly quenched by interaction with regular oxicomplexes and it produces only fast components. Such additional luminescence practically doubles the light yield of scintillation as compared to regular crystals. Three crystal samples with a size of $1.5 \times 1.5 \times 1.5 \mathrm{~cm}^{3}$ have been used for light yield and decay time measurements. The first one was a regular PWO with the standard optimization for the CMS detector. The light yield, measured with a ${ }^{137} \mathrm{Cs}$ source is $15 \mathrm{pe} / \mathrm{MeV}$ and $95 \%$ of the light is emitted in 100ns. The second sample PWO:Mo,La was doped with 500ppm of Molybdenum and 100ppm of Lathanum. The corresponding light yield is $56 \mathrm{pe} / \mathrm{MeV}$ with about $60 \%$ of the light emitted in 100ns. The third sample on the other hand PWO:Tb was doped with $100 \mathrm{ppm}$ of Terbium and a light yield of $27 \mathrm{pe} / \mathrm{MeV}$ was measured with $98 \%$ of the light produced in the first $100 \mathrm{~ns}$. Fig 3 shows the ${ }^{137} \mathrm{Cs}$ light yield spectra of PWO:Mo,La and PWO:Tb. In the same conditions the PWO regular spectrum only shows a shoulder on the background.
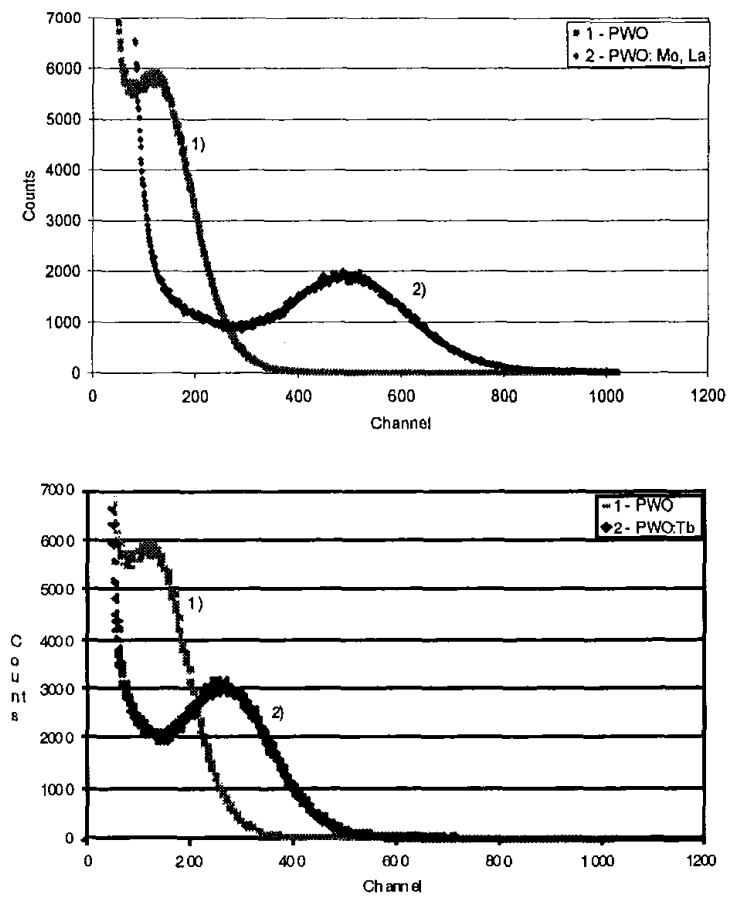

Fig. $3{ }^{137} \mathrm{Cs}$ spectra for PWO'Mo,La and PWO:Tb 


\section{CONCLUSIONS}

After 4 years of intensive R\&D the Bogoroditsk TechnoChemical Plant in Russia has started the preproduction of $600023 \mathrm{~cm}$ long PWO crystals for the CMS electromagnetic calorimeter. An optimisation on crystal growth, based on a proper tuning of the raw material and co-doping with Niobium and Yttrium has led to a significant and consistent improvement of radiation hardness properties of PWO crystals. Similarly a significant improvement of the light yield with extremely fast timing properties has been obtained by doping PWO with $\mathrm{Tb}^{3+}$ ions.

The systematic R\&D effort on scintillating crystals for High Energy Physics experiments has also benefited to other fields. The understanding of some fundamental scintillation mechanisms like energy transfer, interaction with the lattice, and relaxation process, has led to the development of a new, class of fast and efficient scintillators for medical imaging devices. Among them, Lutetium Aluminate Perovskite (LuAP) is in the phase of engineering production technology by the Crystal Clear Collaboration.

\section{ACKNOWLEDGEMENTS}

The authors are particularly grateful to all the people (physicists, engineers and technicians) who have contributed to this effort. The technical staff of the Bogoroditsk Plant must be particularly acknowledged for their flexibility in providing large number of crystals grown in different conditions on our work. We wish also to express our gratitude to all our colleagues from the CMS-ECAL and Crystal Clear for fruitful collaboration and discussions.

\section{REFERENCES}

[1] Alkali halide scintillation counters, R. Hofstadter, Phys. Rev., Vol74, 1948, p100

[2] Luminescence of $\mathrm{Bi} 4 \mathrm{Ge} 3 \mathrm{O} 12$ : spectral and decay properties, M.J. Weber and R.R. Monchamp, J. Appl. Phys., vol. 44, pp 5495-5499, 1973

[3] L3 collaboration: B. Adeva et al., The construction of the L3 experiment, NIM A289 (1990) 35-102

[4] Crystal Clear Collaboration : E. Auffray et al., R\&D Proposal for the study of new fast and radiation hard scintillators for calorimetry at LHC, CERN / DRDC P27 / 91-15, project RD18

[5] Crystal Clear Collaboration: E. Auffray et al., Development of detectors, based on inorganic scintillators, for basic research and applications, The Crystal Clear Project, CERN/ETT status report, Sept 1999, with 261 references

[6] L.L. Nagornaya et al, Proceedings of Crystal 2000 conference, Chamonix, France, Sept. 1992, p367

M. Kobayashi et al, Proceedings of Crystal 2000 conference, Chamonix, France, Sept. 1992, p375

V.G. Boryshevsky et al., NIM A 322 (1992) 231-237
[7] CMS Technical proposal, CERN/LHCC 94-38

The Electromagnetic Calorimeter Project Technical Design Report, CERN/LHCC 97-33

[8] P. Lecoq et al., Lead Tungstate (PbWO4) scintillators for LHC EM calorimetry, NIM A365 (1995) p291

[9] P. Lecoq, CMS-LHCC report 1996-146

[10] E. Auffray et al., Improvement of several properties of lead tungstate crystals with different doping ions, CMS Note 97/54, NIM A 402 (1998) p75

[11] A.N. Annenkov et al., Systematic study of the PbWO4 crystal short term instability under irradiation, CMS Note 97-55, Radiation Measurements Vol29, p27

[12] Kobayashi et al., Improvement of PWO radiation hardness with La doping, KEK report 1997-12

[13] A. Annenkov et al., Suppression of the radiation damage in Lead Tungstate scintillation crystals, 28 Sept98, NIM A426 (1999) $486-490$

[14] M. Kobayashi et al., NIM A 434 (1999) 412-423

[15] M. Korzhik et al., Cerium-doped Lutetium based single crystal scintillators , Proceedings of SCINT95 p355, International Conference on Inorganic Scintillators and their Applications, Delft, TheNetherlands, August 1995

[16] C. Dujardin et al., Luminescence properties and scintillation mechanisms of cerium- and praseodynium-doped lutetium orthoaluminate, J. Phys. Condens. Matter 9 (1997) 5229-5243

[17] J.Chval et al., Development of new mixed $\operatorname{Lu}_{x}\left(\mathrm{RE}^{3+}\right)_{1-x} \mathrm{AP}$ : Ce scintillators $\left(\mathrm{RE}^{3+}=\mathrm{Y}^{3+}\right.$ or $\left.\mathrm{Gd}^{3+}\right)$. Comparison with other Ce-doped or intrinsic scintillating crystals, NIM A 443 (2000) 331-341 